Maltepe Journal of Mathematics

ISSN:2667-7660, URL:HTTP://DERGIPARK.ORG.TR/TR/PUB/MJM

Volume III Issue 2 (2021), PAges 60-73. Doi:HtTPs://DOI.ORG/10.47087/MJM.752726

\title{
THE PATHWAY INTEGRAL OPERATOR INVOLVING EXTENSION OF K-BESSEL-MAITLAND FUNCTION
}

\author{
MOIN AHMAD*, SAURABH PORWAL** \\ *DEPARTMENT OF MATHEMATICS, \\ SHREE KRISHNA EDUCATIONAL INSTITUTE, \\ AHMAD NAGAR SITAPUR-261125, (U.P.), INDIA, 9807480087, ORCID ID: \\ 0000-0002-1482-8197 \\ **DEPARTMENT OF MATHEMATICS, \\ RAM SAHAI GOVERNMENT DEGREE COLLEGE, \\ BAIRI-SHIVRAJPUR, KANPUR-209205, (U.P.), INDIA, 9415937193, ORCID ID: \\ 0000-0003-0847-3550
}

\begin{abstract}
In the present paper, we establish generalized extension of kBessel-Maitland function involving pathway integral operator. We obtain certain composition formulas with pathway fractional integral operators. Further more, Some interesting special cases involving Bessel functions, generalized Bessel functions, generalized Mittag-Leffer functions, generalized k-MittagLeffer functions are deduced.
\end{abstract}

\section{Introduction}

The study of special functions play an important role in Mathematics, Physics, Chemistry, Biology, Engineering and applied Sciences. It has a wide application of almost all branches of Science and technology. The Bessel-Maitland function [10, 28, is denoted by $J_{\nu}^{\mu}(z)$ and is defined as follows:

$$
\mathbf{J}_{\nu}^{\mu}(z)=\sum_{n=0}^{\infty} \frac{(-z)^{n}}{n ! \Gamma(n \mu+\nu+1)} .
$$

The theory of Bessel functions is intimately connected with the theory of certain types of differential equations. A detail account of applications of Bessel functions are given in the book of Watson [27.

Now, Singh et al. [25] introduced and investigate of the following generalization of Bessel-Maitland function as follows:

$$
\mathbf{J}_{\nu, \tau}^{\mu, q}(z)=\sum_{n=0}^{\infty} \frac{(\tau)_{q n}(-z)^{n}}{n ! \Gamma(n \mu+\nu+1)},
$$

2010 Mathematics Subject Classification. 42C05,26A33,33C45.

Key words and phrases. Bessel-Maitland function, fractional calculus, pathway integral. (C)2019 Maltepe Journal of Mathematics.

Submitted on June 14th, 2021. Published on October 30 th, 2021.

Communicated by Metin BASARIR . 
THE PATHWAY INTEGRAL OPERATOR INVOLVING EXTENSION OF K-BESSEL-MAITLAND FUNCTION

where $\mu, \nu, \tau \in \mathbb{C} ; \Re(\mu) \geq 0, \Re(\nu) \geq-1, \Re(\tau) \geq 0$, and $q \in(0,1) \bigcup \mathbb{N}$ and $(\tau)_{q n}=\frac{\Gamma(\tau+q n)}{\Gamma(\tau)}$ denotes the generalized Pochhammer symbol (see Rainville [21]).

Furthermore, Ghayasuddin et al. [7] investigate a new extension of BesselMaitland function as follows:

$$
\mathbf{J}_{\nu, \tau, \zeta}^{\mu, q, p}(z)=\sum_{n=0}^{\infty} \frac{(\tau)_{q n}(-z)^{n}}{\Gamma(n \mu+\nu+1)(\zeta)_{p n}},
$$

where $\mu, \nu, \tau, \zeta \in \mathbb{C} ; \Re(\mu) \geq 0, \Re(\nu) \geq-1, \Re(\tau) \geq 0, \Re(\zeta) \geq 0 ; p, q>0$, and $q<\Re(\alpha)+p$.

Recently, Khan et al. 9] consider a new generalized Bessel-Maitland function which is defined as:

$$
\mathbf{J}_{\alpha, \beta, \nu, \sigma, \zeta, p}^{\mu, \rho, \tau, q}(z)=\sum_{n=0}^{\infty} \frac{(\mu)_{\rho n}(\tau)_{q n}(-z)^{n}}{\Gamma(n \beta+\alpha+1)(\zeta)_{p n}(\nu)_{n \sigma}},
$$

where $\alpha, \beta, \mu, \rho, \nu, \tau, \zeta \in \mathbb{C} ; \Re(\alpha)>0, \Re(\beta)>0, \Re(\rho)>0, \Re(\mu) \geq 0, \Re(\nu) \geq$ $-1, \Re(\tau) \geq 0, \Re(\zeta) \geq 0 ; p, q>0$, and $q<\Re(\alpha)+p$.

In this paper, we consider a new extension of generalized k-Bessel-Maitland function which is defined as:

$$
\mathbf{J}_{k, \alpha, \beta, \nu, \sigma, \zeta, p}^{\mu, \rho, \tau, q}(z)=\sum_{n=0}^{\infty} \frac{(\mu)_{\rho n, k}(\tau)_{q n, k}(-z)^{n}}{\Gamma_{k}(n \beta+\alpha+1)(\delta)_{p n, k}(\nu)_{n \sigma, k}},
$$

where $k, \alpha, \beta, \mu, \rho, \nu, \tau, \zeta \in \mathbb{C} ; \Re(\alpha)>0, \Re(\beta)>0, \Re(\rho)>0, \Re(\mu) \geq 0, \Re(\nu) \geq$ $-1, \Re(\gamma) \geq 0, \Re(\delta) \geq 0 ; p, q>0$, and $q<\Re(\alpha)+p$.

\subsection{Relation with Mittag-Leffler function.}

(1) If we put $\alpha$ by $\alpha-1$ in 1.5 , we get the following result

$$
\mathbf{J}_{k, \alpha-1, \beta, \nu, \sigma, \zeta, p}^{\mu, \rho, \tau, q}(-x)=E_{k, \alpha, \beta, \nu, \sigma, \zeta, p}^{\mu, \rho, \tau, q}(x),
$$

where $E_{k, \alpha, \beta, \nu, \sigma, \zeta, p}^{\mu, \rho, \tau, q}(x)$ is the Mittag-Leffler function defined by Khan and Ahmad [8].

(2) If we put $\mu=\nu=\sigma=\rho=k=1$ and replacing $\alpha$ by $\alpha-1$ in $(1.5)$, we get

$$
\mathbf{J}_{\alpha-1, \beta, 1,1, \delta, p}^{1,1, \gamma, q}(-x)=E_{\alpha, \beta, p}^{\zeta, \tau, q}(x),
$$

where $E_{\alpha, \beta, p}^{\zeta, \tau, q}(x)$ is the Mittag-Leffler function defined by Salim and Faraz 23 .

(3) If we put $\mu=\nu=\sigma=\rho=\zeta=p=1$ and replacing $\alpha$ by $\alpha-1$ in $(1.5)$, we get

$$
\mathbf{J}_{k, \alpha-1, \beta, 1,1,1,1}^{1,1, \zeta, q}(-x)=E_{k, \alpha, \beta}^{\tau, q}(x),
$$

where $E_{k, \alpha, \beta}^{\tau, q}(x)$ is the k-Mittag-Leffler function defined by Chand et al. 4]. 
(4) If we put $\mu=\nu=\sigma=\rho=\zeta=p=k=1$ and replacing $\alpha$ by $\alpha-1$ in (1.5), we get

$$
\mathbf{J}_{\alpha-1, \beta, 1,1,1,1}^{1,1, \gamma, q}(-x)=E_{\alpha, \beta}^{\tau, q}(x),
$$

where $E_{\alpha, \beta}^{\tau, q}(x)$ is the Mittag-Leffler function defined by Shukla and Prajapati [26].

(5) If we put $\mu=\nu=\sigma=\rho=\zeta=1$ and replacing $\alpha$ by $\alpha-1$ in 1.5), we get

$$
\mathbf{J}_{\alpha-1, \beta, 1,1,1,1}^{1,1, \tau, \zeta}(-x)=E_{\alpha, \beta}^{\tau, \zeta}(x),
$$

where $E_{\alpha, \beta}^{\tau, q}(x)$ is the Mittag-Leffler function defined by Salim [24].

(6) If we put $\mu=\nu=\sigma=\rho=\zeta=p=q=1$ and replacing $\alpha$ by $\alpha-1$ in (1.5), we get

$$
\mathbf{J}_{k, \alpha-1, \beta, 1,1,1,1}^{1,1, \tau}(-x)=E_{k, \alpha, \beta}^{\tau}(x),
$$

where $E_{k, \alpha, \beta}^{\tau}(x)$ is the k-Mittag-Leffler function defined by Dorrego and Cerutti [6].

(7) If we put $\mu=\nu=\sigma=\rho=\zeta=p=q=k=1$ and replacing $\alpha$ by $\alpha-1$ in (1.5), we get

$$
\mathbf{J}_{\alpha-1, \beta, 1,1,1,1}^{1,1, \tau}(-x)=E_{\alpha, \beta}^{\tau}(x),
$$

where $E_{\alpha, \beta}^{\tau}(x)$ is the Mittag-Leffler function defined by Prabhakar [22].

(8) If we put $\mu=\nu=\sigma=\rho=\zeta=\tau=p=q=k=1$ and replacing $\alpha$ by $\alpha-1$ in 1.5$)$, we get

$$
\mathbf{J}_{\alpha-1, \beta, 1,1,1,1}^{1,1,1}(-x)=E_{\alpha, \beta}(x),
$$

where $E_{\alpha, \beta}(x)$ is the Mittag-Leffler function defined by Wiman [28].

(9) If we put $\mu=\nu=\sigma=\rho=\zeta=\tau=p=q=k=1, \alpha=0$ and replacing $\alpha$ by $\alpha-1$ in 1.5 , we get

$$
\mathbf{J}_{0, \beta, 1,1,1,1}^{1,1,1}(-x)=E_{\beta}(x),
$$

where $E_{\beta}(x)$ is the Mittag-Leffler function defined by Mittag-Leffler [16].

We investigate some special cases of the generalized Bessel Maitland function (1.3) by particular values to the parameters $\mu, \nu, \delta, \gamma, p, q$.

Now, we recall the classical Beta function denoted by $B(a, b)$ and is defined as

$$
B(a, b)=\int_{0}^{1} t^{a-1}(1-t)^{b-1} d t=\frac{\Gamma(a) \Gamma(b)}{\Gamma(a+b)},(\Re(a)>0, \Re(b)>0) .
$$

(see [21, and also see [10]). The integral representation of the k-Gamma function is given as:

$$
\Gamma_{k}(z)=k^{\frac{z}{k}-1} \Gamma\left(\frac{z}{k}\right)=\int_{0}^{\infty} e^{\frac{-t^{k}}{k}} t^{z-1} d t,
$$

$k \in \mathbb{R}, z \in \mathbb{C}$, 
THE PATHWAY INTEGRAL OPERATOR INVOLVING EXTENSION OF K-BESSEL-MAITLAND FUNCTIOA

and $\mathrm{k}$-Beta function is defined as:

$$
B_{k}(x, y)=\frac{1}{k} \int_{0}^{1} t^{\frac{x}{k}-1}(1-t)^{\frac{y}{k}-1} d t=\frac{\Gamma_{k}(x) \Gamma_{k}(y)}{\Gamma_{k}(x+y)}, x>0, y>0 .
$$

The generalized Wright function represented as follows [29, 30, 31]:

$$
\begin{gathered}
\left.{ }_{p} \Psi_{q}\left[\begin{array}{c}
\left(\alpha_{1}, A_{1}\right), \ldots,\left(\alpha_{p}, A_{p}\right) ; \\
\left(\beta_{1}, B_{1}\right), \ldots,\left(\beta_{p}, B_{p}\right) ;
\end{array}\right]={ }_{p} \Psi_{q}\left(\left(\alpha_{j}, A_{j}\right)\right)_{1, p} ;\left(\beta_{j}, B_{j}\right)_{1, q} ; z\right) \\
=\sum_{n=0}^{\infty} \frac{\Gamma\left(\alpha_{1}+n A_{1}\right) \ldots, \Gamma\left(\alpha_{p}+n A_{p}\right)}{\Gamma\left(\beta_{1}+n B_{1}\right) \ldots, \Gamma\left(\beta_{p}+n B_{p}\right)} \frac{z^{n}}{n !} .
\end{gathered}
$$

In 1961, MacRobert [1] investigate the following interesting result which is given below:

$$
\int_{0}^{1} t^{\alpha-1}(1-t)^{\beta-1}[a t+b(1-t)]^{-\alpha-\beta} d t=\frac{1}{a^{\alpha} b^{\beta}} \frac{\Gamma(\alpha) \Gamma(\beta)}{\Gamma(\alpha+\beta)},
$$

where $a$ and $b$ are non zero constants such that the expression $a t+b(1-t)$, for $0 \leq t \leq 1$, is non zero, provided $\Re(\alpha)>0, \Re(\beta)>0$.

In this paper, we further apply the following useful result which is given below:

$$
\int_{0}^{1} t^{\frac{\alpha}{k}-1}(1-t)^{\frac{\beta}{k}-1}[a t+b(1-t)]^{\frac{-\alpha-\beta}{k}} d t=\frac{1}{a^{\frac{\alpha}{k}} b^{\frac{\beta}{k}}} \frac{k \Gamma_{k}(\alpha) \Gamma_{k}(\beta)}{\Gamma_{k}(\alpha+\beta)},
$$

where $a$ and $b$ are non zero constants such that the expression $a t+b(1-t)$, for $0 \leq t \leq 1$, is non zero, provided $\Re(\alpha)>0, \Re(\beta)>0$.

It is easy to see that for $k=1$ the equation 1.20 reduces to known result 1.19 .

Recently, by using the pathway idea of Mathai [13] and developed further by Mathai and Haubold [14, 15], Nair [17, we introduce a pathway fractional integral operator which is given below.

Suppose $f(x) \in L(a, b), \eta \in \mathbb{C}, \Re(\eta)>0, a>0$ and the pathway parameter $\alpha<1$ as (cf. 2]), then

$$
\left(P_{0+}^{(\eta, \alpha)} f\right)(x)=x^{\eta} \int_{0}^{\left[\frac{x}{a(1-\alpha)}\right]}\left[1-\frac{a(1-\alpha) t}{x}\right]^{\frac{\eta}{(1-\alpha)}} f(t) d t .
$$

For a real scalar $\alpha$, the pathway model for scalar random variables is represented by the following probability density function (p.d.f.):

$$
f(x)=c|x|^{\gamma-1}\left[1-a(1-\alpha)|x|^{\delta}\right]^{\frac{\beta}{(1-\alpha)}}
$$


provided that $-\infty<x<\infty, \delta>0, \beta \geq 0,\left[1-a(1-\alpha)|x|^{\delta}\right]>0$ and $\gamma>0$, where $c$ is the normalizing constant and $\alpha$ is called the pathway parameter,

$$
\begin{aligned}
c & =\frac{1}{2} \frac{\delta(a(1-\alpha))^{\frac{\gamma}{\delta}} \Gamma\left(\frac{\gamma}{\delta}+\frac{\beta}{(1-\alpha)}+1\right)}{\Gamma\left(\frac{\gamma}{\delta}\right) \Gamma\left(\frac{\beta}{(1-\alpha)}+1\right)}, \text { for } \alpha<1 \\
& =\frac{1}{2} \frac{\delta(a(1-\alpha))^{\frac{\gamma}{\delta}} \Gamma\left(\frac{\beta}{(1-\alpha)}\right)}{\Gamma\left(\frac{\gamma}{\delta}\right) \Gamma\left(\frac{\beta}{(1-\alpha)}-\frac{\gamma}{\delta}\right)}, \text { for } \frac{1}{1-\alpha}-\frac{\gamma}{\delta}>0, \alpha>1 \\
& =\frac{1}{2} \frac{(a \beta)^{\frac{\gamma}{\delta}}}{\Gamma\left(\frac{\gamma}{\delta}\right)}, \alpha \rightarrow 1 .
\end{aligned}
$$

For $\alpha<1$, it is a finite range density with $\left[1-a(1-\alpha)|x|^{\delta}\right]>0$ and 1.21 ) remains in the extended generalized type- 1 beta family. The Pathway density in (1.21), for $\alpha<1$, includes the extended type-1 beta density, the triangular density, the uniform density and many other p.d.f's. [2]. For $\alpha>1$,

$$
f(x)=c|x|^{\gamma-1}\left[1+a(1-\alpha)|x|^{\delta}\right]^{-\frac{\beta}{1-\alpha}},
$$

provided that $-\infty<x<\infty, \delta>0, \beta \geq 0$ and $\alpha>0$ which is extended generalized type- 2 modal for real $x$. It includes the type-2 beta density, the $F$ density, the student-t density, the cauchy density and many more. For instance, $\alpha>1$, writing $(1-\alpha)=-(\alpha-1)$ gives:

$$
\left(P_{0+}^{(\eta, \alpha)} f\right)(x)=x^{\eta} \int_{0}^{\left[\frac{x}{-a(1-\alpha)}\right]}\left[1+\frac{a(\alpha-1) t}{x}\right]^{-\frac{\eta}{(\alpha-1)}} f(t) d t .
$$

For more basic details about pathway integral operator, one may refer [1, 2, 18, 19, 20.

\section{MAin Results}

The pathway integral operator of k-Bessel-Maitland function is given in the following theorems.

Theorem 2.1. Let $k \in \mathcal{R}, \alpha, \beta, \tau, \zeta, \mu, \nu, \rho, \sigma \in \mathcal{C}, \Re(\alpha)>0, \Re(\beta)>0, \Re(\tau)>$ $0, \Re(\zeta)>0, \Re(\mu)>0, \Re(\nu)>0, \Re(\rho)>0, \Re(\sigma)>0, p, q>0$ and $q \leq \Re(\alpha)+p, \eta \in$ $C, \Re\left(\frac{\eta}{1-\xi}\right)>-1, \lambda>1, w>R$.

$$
P_{0+}^{(\eta, \lambda)}\left[t^{\frac{\beta}{k}-1} \mathbf{J}_{k, \alpha, \beta, \nu, \sigma, \zeta, p}^{\mu, \rho, q}\left(w t^{\frac{\alpha}{k}}\right)\right](x)=\frac{x^{\eta+\frac{\beta}{k}} \Gamma\left(\frac{\eta}{(1-\lambda)}+1\right)}{\left(a(1-\lambda)^{\frac{\beta}{k}} k^{\frac{\alpha+1}{k}-1}\right.} \mathbf{J}_{k, \alpha, \beta+k\left(\frac{\eta}{1-\lambda}\right), \nu, \sigma, \zeta, p}^{\mu, \rho, q}\left(w\left(\frac{x}{a(1-\lambda)}\right)^{\frac{\alpha}{k}}\right) .
$$

Proof. On taking L.H.S. of Theorem 2.1 and then expanding the definition of generalized k-Bessel-Maitland function $\mathbf{J}_{k, \alpha, \beta, \nu, \sigma, \zeta, p}^{\mu, \rho, \tau, q}\left(w t^{\frac{\alpha}{k}}\right)$, by using 1.18 we obtain:

$$
P_{0+}^{(\eta, \lambda)}\left[t^{\frac{\beta}{k}-1} J_{k, \alpha, \beta, \nu, \sigma, \zeta, p}^{\mu, \rho, \tau, q}\left(w t^{\frac{\alpha}{k}}\right)\right](x)
$$


THE PATHWAY INTEGRAL OPERATOR INVOLVING EXTENSION OF K-BESSEL-MAITLAND FUNCTIOAS

$$
\begin{gathered}
=x^{\eta} \int_{0}^{\left[\frac{x}{a(1-\lambda)}\right]} t^{\frac{\beta}{k}-1}\left[1-\frac{a(1-\lambda) t}{x}\right]^{\frac{\eta}{(1-\lambda)}} J_{k, \alpha, \beta, \nu, \sigma, \zeta, p}^{\mu, \rho, q, q}\left(w t^{\frac{\alpha}{k}}\right) d t, \\
=x^{\eta} \int_{0}^{\left[\frac{x}{a(1-\lambda)}\right]} t^{\frac{\beta}{k}-1}\left[1-\frac{a(1-\lambda) t}{x}\right]^{\frac{\eta}{(1-\lambda)}} \sum_{n=0}^{\infty} \frac{(\mu)_{\rho n, k}(\tau)_{q n, k}\left(-w t^{\frac{\alpha}{k}}\right)^{n}}{\Gamma_{k}(n \beta+\alpha+1)(\zeta)_{p n, k}(\nu)_{n \sigma, k}} d t,
\end{gathered}
$$

Interchanging the integration and summation under the suitable convergence condition, we obtain

$$
=x^{\eta} \sum_{n=0}^{\infty} \frac{(\mu)_{\rho n, k}(\tau)_{q n, k}(-w)^{n}}{\Gamma_{k}(n \beta+\alpha+1)(\zeta)_{p n, k}(\nu)_{n \sigma, k}} \int_{0}^{\left[\frac{x}{a(1-\lambda)}\right]} t^{\frac{\beta}{k}+\frac{n \alpha}{k}-1}\left[1-\frac{a(1-\lambda) t}{x}\right]^{\frac{\eta}{(1-\lambda)}} d t,
$$

Now, interchanging the inner integral by beta function formula 1.12 , we get

$$
\begin{gathered}
=x^{\eta} \sum_{n=0}^{\infty} \frac{(\mu)_{\rho n, k}(\tau)_{q n, k}(-w)^{n}}{\Gamma_{k}(n \beta+\alpha+1)(\zeta)_{p n, k}(\nu)_{n \sigma, k}} \int_{0}^{1} u^{\frac{\beta}{k}+\frac{n \alpha}{k}-1}(1-u)^{\frac{\eta}{(1-\lambda)}}\left(\frac{x}{a(1-\lambda)}\right) \\
\times\left(\frac{x}{a(1-\lambda)}\right)^{\frac{\beta}{k}+\frac{n \alpha}{k}-1} d u,
\end{gathered}
$$

again applying the Beta function formula, we have

$$
=\frac{x^{\eta+\frac{\beta}{k}}}{\left(a(1-\lambda)^{\frac{\beta}{k}}\right.} \sum_{n=0}^{\infty} \frac{(\mu)_{\rho n, k}(\tau)_{q n, k}(-w)^{n} x^{\frac{n \alpha}{k}}}{\Gamma_{k}(n \beta+\alpha+1)(\zeta)_{p n, k}(\nu)_{n \sigma, k}} \frac{\Gamma\left(\frac{\eta}{(1-\lambda)}+1\right) \Gamma\left(\frac{\beta}{k}+\frac{n \alpha}{k}\right)}{\Gamma\left(\frac{\eta}{(1-\lambda)}+\frac{\beta}{k}+\frac{n \alpha}{k}+1\right)} \frac{1}{(a(1-\lambda))^{\frac{n \alpha}{k}}} .
$$

Now, using the result,

$$
\Gamma_{k}(\lambda)=k^{\frac{\lambda}{k}-1} \Gamma\left(\frac{\lambda}{k}\right)
$$

we get,

$$
\begin{gathered}
=\frac{x^{\eta+\frac{\beta}{k}} \Gamma\left(\frac{\eta}{(1-\lambda)}+1\right)}{\left(a(1-\lambda)^{\frac{\beta}{k}}\right.} \sum_{n=0}^{\infty} \frac{(\mu)_{\rho n, k}(\tau)_{q n, k}(-w)^{n} x^{\frac{n \alpha}{k}}}{k^{\frac{n+\alpha+\alpha+1}{k}-1} \Gamma\left(\frac{n \beta+\alpha+1}{k}\right)(\zeta)_{p n, k}(\nu)_{n \sigma, k}} \frac{\Gamma\left(\frac{\beta}{k}+\frac{n \alpha}{k}\right)}{\Gamma\left(\frac{\eta}{(1-\lambda)}+\frac{\beta}{k}+\frac{n \alpha}{k}+1\right)} \frac{1}{(a(1-\lambda))^{\frac{n \alpha}{k}}}, \\
=\frac{x^{\eta+\frac{\beta}{k}} \Gamma\left(\frac{\eta}{(1-\lambda)}+1\right)}{\left(a(1-\lambda)^{\frac{\beta}{k}} k^{\frac{\alpha+1}{k}-1}\right.} \mathbf{J}_{k, \alpha, \beta+k\left(\frac{\eta}{1-\lambda}\right), \nu, \sigma, \zeta, p}^{\mu, \rho, q}\left(w\left(\frac{x}{a(1-\lambda)}\right)^{\frac{\alpha}{k}}\right),
\end{gathered}
$$

which is our desired result 2.1).

Thus, the proof of Theorem 2.1] is complete. 
Corollary 2.2. If we put $\tau=q=1, \nu=\sigma=p=1$ in Theorem 2.1, then we get the result corresponding result of Nisar et al. [19] as:

$$
P_{0+}^{(\eta, \lambda)}\left[t^{\frac{\beta}{k}-1} \mathbf{J}_{k, \alpha, \beta, 1,1, \zeta, 1}^{\mu, \rho, 1,1}\left(w t^{\frac{\alpha}{k}}\right)\right](x)=\frac{x^{\eta+\frac{\beta}{k}} \Gamma\left(\frac{\eta}{(1-\lambda)}+1\right)}{\left(a(1-\lambda)^{\frac{\beta}{k}} k^{\frac{\alpha+1}{k}-1}\right.} \mathbf{J}_{k, \alpha, \beta+k\left(\frac{\eta}{1-\lambda}\right), 1,1, \zeta, 1}^{\mu, 1,1}\left(-w\left(\frac{x}{a(1-\lambda)}\right)^{\frac{\alpha}{k}}\right) .
$$

Corollary 2.3. If we put $\tau=q=1, \nu=\sigma=p=\zeta=k=1$ in Theorem 2.1, then we obtain the corresponding result of Nair [17] as:

$$
P_{0+}^{(\eta, \lambda)}\left[t^{\beta-1} \mathbf{J}_{1, \alpha, \beta, 1,1,1,1}^{\mu, \rho, 1,1}\left(w t^{\frac{\alpha}{k}}\right)\right](x)=\frac{x^{\eta+\beta} \Gamma\left(\frac{\eta}{(1-\lambda)}+1\right)}{(a(1-\lambda))^{\beta}} \mathbf{J}_{1, \alpha, \beta+1\left(\frac{\eta}{1-\lambda}\right), 1,1,1,1}^{\mu, \rho, 1}\left(w\left(\frac{x}{a(1-\lambda)}\right)^{\alpha}\right) .
$$

Theorem 2.4. Let $k \in \mathcal{R}, \alpha, \beta, \tau, \zeta, \mu, \nu, \rho, \sigma \in \mathcal{C}, \Re(\alpha)>0, \Re(\beta)>0, \Re(\tau)>$ $0, \Re(\zeta)>0, \Re(\mu)>0, \Re(\nu)>0, \Re(\rho)>0, \Re(\sigma)>0, p, q>0$ and $q \leq \Re(\alpha)+p, \eta \in$ $C, \Re\left(\frac{\eta}{1-\xi}\right)>-1, \lambda>1, w>R$

$P_{0+}^{(\eta, \lambda)}\left[t^{\frac{\beta}{k}-1} \mathbf{J}_{k, \alpha, \beta, \rho, \tau, \sigma, \zeta, p}^{\mu, q}\left(w t^{\frac{\alpha}{k}}\right)\right](x)=\frac{x^{\eta+\frac{\beta}{k}+1} \Gamma\left(1-\frac{\eta}{(\lambda-1)}\right)}{\left(-a(1-\lambda)^{\frac{\beta}{k}} k^{\frac{\alpha+1}{k}-1}\right.} \mathbf{J}_{k, \alpha, \beta, \beta, k\left(n \alpha+k-\frac{\eta}{\lambda-1}\right), \nu, \sigma, \zeta, p}^{\mu, q}\left(w\left(\frac{x}{-a(\lambda-1)}\right)^{\frac{\alpha}{k}}\right)$.

Proof. On taking L.H.S of 2.5 and applying the definition 1.5 and 1.24 , we obtain

$$
\begin{aligned}
& P_{0+}^{(\eta, \lambda)}\left[t^{\frac{\beta}{k}-1} \mathbf{J}_{k, \alpha, \beta, \nu, \sigma, \zeta, p}^{\mu, \tau, \tau}\left(w t^{\frac{\alpha}{k}}\right)\right](x) \\
& =x^{\eta} \int_{0}^{\left[\frac{x}{-a(1-\lambda)}\right]} t^{\frac{\beta}{k}-1}\left[1+\frac{a(\lambda-1) t}{x}\right]^{\frac{\eta}{-(\lambda-1)}} J_{k, \alpha, \beta, \nu, \sigma, \zeta, p}^{\mu, \rho, \tau, q}\left(w t^{\frac{\alpha}{k}}\right) d t, \\
& =x^{\eta} \int_{0}^{\left[\frac{x}{-a(1-\lambda)}\right]} t^{\frac{\beta}{k}-1}\left[1+\frac{a(\lambda-1) t}{x}\right]^{\frac{\eta}{-(\lambda-1)}} \sum_{n=0}^{\infty} \frac{(\mu)_{\rho n, k}(\tau)_{q n, k}\left(-w t^{\frac{\alpha}{k}}\right)^{n}}{\Gamma_{k}(n \beta+\alpha+1)(\zeta)_{p n, k}(\nu)_{n \sigma, k}} d t .
\end{aligned}
$$

Interchanging the integration and summation under the suitable convergence condition, we obtain

$$
=x^{\eta} \sum_{n=0}^{\infty} \frac{(\mu)_{\rho n, k}(\tau)_{q n, k}(-w)^{n}}{\Gamma_{k}(n \beta+\alpha+1)(\zeta)_{p n, k}(\nu)_{n \sigma, k}} \int_{0}^{\left[\frac{x}{-a(1-\lambda)}\right]} t^{\frac{\beta}{k}+\frac{n \alpha}{k}-1}\left[1+\frac{a(\lambda-1) t}{x}\right]^{-\frac{\eta}{-(\lambda-1)}} d t .
$$

Now, interchanging the inner integral by beta function formula, we get

$$
=x^{\eta} \sum_{n=0}^{\infty} \frac{(\mu)_{\rho n, k}(\tau)_{q n, k}(-w)^{n}}{\Gamma_{k}(n \beta+\alpha+1)(\zeta)_{p n, k}(\nu)_{n \sigma, k}} \int_{0}^{1} u^{\frac{\beta}{k}+\frac{n \alpha}{k}-1}(1-u)^{\frac{\eta}{(1-\lambda)}}\left(\frac{x}{a(1-\lambda)}\right)
$$


THE PATHWAY INTEGRAL OPERATOR INVOLVING EXTENSION OF K-BESSEL-MAITLAND FUNCTIO

$$
\times\left(\frac{x}{a(1-\lambda)}\right)^{\frac{\beta}{k}+\frac{n \alpha}{k}-1} d u
$$

again applying the beta function formula, we have

$$
=\frac{x^{\eta+\frac{\beta}{k}}}{\left(-a(1-\lambda)^{\frac{\beta}{k}}\right.} \sum_{n=0}^{\infty} \frac{(\mu)_{\rho n, k}(\tau)_{q n, k}(-w)^{n} x^{\frac{n \alpha}{k}}}{\Gamma_{k}(n \beta+\alpha+1)(\zeta)_{p n, k}(\nu)_{n \sigma, k}} \frac{\Gamma\left(1-\frac{v}{(\lambda-1)}\right) \Gamma\left(\frac{\beta}{k}+\frac{n \alpha}{k}\right)}{\Gamma\left(1-\frac{v}{(\lambda-1)}+\frac{\beta}{k}+\frac{n \alpha}{k}\right)} \frac{1}{(-a(\lambda-1))^{\frac{n \alpha}{k}}} .
$$

Now, using the result,

we obtain,

$$
\Gamma_{k}(\lambda)=k^{\frac{\lambda}{k}-1} \Gamma\left(\frac{\lambda}{k}\right)
$$

$$
\begin{gathered}
=\frac{x^{\eta+\frac{\beta}{k}+1} \Gamma\left(1-\frac{\eta}{(\lambda-1)}\right)}{\left(-a(1-\lambda)^{\frac{\beta}{k}+1}\right.} \sum_{n=0}^{\infty} \frac{(\mu)_{\rho n, k}(\tau)_{q n, k}(-w)^{n} x^{\frac{n \alpha}{k}}}{k^{\frac{n \beta+\alpha+1}{k}-1} \Gamma\left(\frac{n \beta+\alpha+1}{k}\right)(\zeta)_{p n, k}(\nu)_{n \sigma, k}} \frac{\Gamma\left(\frac{\beta}{k}+\frac{n \alpha}{k}\right)}{\Gamma\left(1-\frac{\eta}{(1-\lambda)}+\frac{\beta}{k}+\frac{n \alpha}{k}\right)} \frac{1}{(-a(1-\lambda))^{\frac{n \alpha}{k}}} \\
=\frac{x^{\eta+\frac{\beta}{k}+1} \Gamma\left(1-\frac{\eta}{(\lambda-1)}\right)}{\left(-a(1-\lambda)^{\frac{\beta}{k}} k^{\frac{\alpha+1}{k}-1}\right.} \mathbf{J}_{k, \alpha, \beta+k\left(n \alpha+k-\frac{\eta}{\lambda-1}\right), \nu, \sigma, \zeta, p}^{\mu, \rho, q}\left(w\left(\frac{x}{-a(\lambda-1)}\right)^{\frac{\alpha}{k}}\right)
\end{gathered}
$$

which is our desired result 2.5 .

Corollary 2.5. If we put $\tau=q=1, \nu=\sigma=p=1$ in Theorem 2.4, then it reduces to the corresponding result of [16]:

$P_{0+}^{(\eta, \lambda)}\left[t^{\frac{\beta}{k}-1} \mathbf{J}_{k, \alpha, \beta, 1,1, \zeta, 1}^{\mu, \rho, 1,1}\left(w t^{\frac{\alpha}{k}}\right)\right](x)=\frac{x^{\eta+\frac{\beta}{k}+1} \Gamma\left(1-\frac{\eta}{(\lambda-1)}\right)}{\left(-a(1-\lambda)^{\frac{\beta}{k}+1} k^{\frac{\alpha+1}{k}-1}\right.} \mathbf{J}_{k, \alpha, \beta+k\left(n \alpha+k-\frac{\eta}{1-\lambda}\right), 1,1, \zeta, 1}^{\mu, 1,1}\left(w\left(\frac{x}{-a(\lambda-1)}\right)^{\frac{\alpha}{k}}\right)$

Corollary 2.6. If we put $\tau=q=1, \nu=\sigma=p=\zeta=k=1$ in Theorem 2.4, then it reduces to the following result of Nair [17.

$P_{0+}^{(\eta, \lambda)}\left[t^{\beta-1} \mathbf{J}_{1, \alpha, \beta, 1,1,1,1}^{\mu, \rho, 1,1}\left(w t^{\alpha}\right)\right](x)=\frac{x^{\eta+\beta+1} \Gamma\left(1-\frac{\eta}{(1-\lambda)}\right)}{(-a(1-\lambda))^{\beta+1}} \mathbf{J}_{1, \alpha, \beta+1\left(n \alpha+1-\frac{\eta}{\lambda-1}\right), 1,1,1,1}^{\mu, \rho, 1,1}\left(w\left(\frac{x}{a(1-\lambda)}\right)^{\alpha}\right)$

Theorem 2.7. Let $k \in \mathcal{R}, \alpha, \beta, v, \zeta, \mu, \nu, \rho, \sigma, \lambda, \tau \in \mathcal{C}, \Re(\alpha)>-1, \Re(\beta)>0, \Re(v)>$ $0, \Re(\zeta)>0, \Re(\mu)>0, \Re(\nu)>0, \Re(\rho)>0, \Re(\sigma)>0, \Re(\lambda)>0, \Re(\tau)>0, p, q>0$ and $q \leq \Re(\alpha)+p$.

$$
\begin{aligned}
& \int_{0}^{1} t^{\frac{v}{k}-1}(1-t)^{\frac{\xi}{k}-1}[a t+b(1-t)]^{\frac{-v-\xi}{k}} \mathbf{J}_{k, \alpha, \beta, \nu, \sigma, \zeta, p}^{\mu, \rho, \tau, q}\left[\frac{2 a b t(1-t)}{(a t+b(1-t))^{2}}\right]^{\frac{1}{k}} d t \\
= & \frac{\Gamma_{k}(\zeta) \Gamma_{k}(\mu)}{\Gamma_{k}(\tau) \Gamma_{k}(\mu) a^{v} b^{\lambda}} \sum_{s=0}^{\infty} \frac{\Gamma_{k}(\mu+s \rho) \Gamma_{k}(\gamma+s q)(-2)^{\frac{s}{k}} a^{\frac{s}{k}} b^{\frac{s}{k}}}{\Gamma_{k}(s \beta+\alpha+1) \Gamma_{k}(\zeta+p s) \Gamma_{k}(\nu+s \sigma) \Gamma} \frac{\Gamma_{k}(v+s) \Gamma_{k}(\lambda+s)}{\Gamma_{k}(v+\lambda+2 s)} .
\end{aligned}
$$


Proof. On taking L.H.S. of Theorem 2.7, using the definition of generalized kBessel-Maitland function (1.5) and (1.17), we obtain

$$
\begin{aligned}
& \int_{0}^{1} t^{\frac{v}{k}-1}(1-t)^{\frac{\xi}{k}-1}[a t+b(1-t)]^{\frac{-\tau-\xi}{k}} \mathbf{J}_{k, \alpha, \beta, \nu, \sigma, \zeta, p}^{\mu, \rho, q}\left[\frac{2 a b t(1-t)}{(a t+b(1-t))^{2}}\right]^{\frac{1}{k}} d t, \\
& =\int_{0}^{1} t^{\frac{v}{k}-1}(1-t)^{\frac{\xi}{k}-1}[a t+b(1-t)]^{\frac{-v-\xi}{k}} \sum_{s=0}^{\infty} \frac{(\mu)_{\rho s, k}(\tau)_{q s, k}}{\Gamma_{k}(s \beta+\alpha+1)(\zeta)_{p s, k}(\nu)_{s \sigma, k}} \frac{(-2)^{\frac{s}{k}}(a b)^{\frac{s}{k}} t^{\frac{s}{k}}(1-t)^{\frac{s}{k}}}{(a t+b(1-t))^{\frac{2 s}{k}}} d t, \\
& =\sum_{s=0}^{\infty} \frac{(\mu)_{\rho s, k}(\tau)_{q s, k}}{\Gamma_{k}(s \beta+\alpha+1)(\zeta)_{p s, k}(\nu)_{s \sigma, k}}(-2)^{\frac{s}{k}}(a b)^{\frac{s}{k}} \int_{0}^{1} t^{\frac{v+s}{k}-1}(1-t)^{\frac{\xi+s}{k}-1}[a t+b(1-t)]^{\frac{-v-\xi-2 s}{k}} d t,
\end{aligned}
$$

by using the integral (1.17), we obtain

$$
\begin{gathered}
=\sum_{s=0}^{\infty} \frac{(\mu)_{\rho s, k}(\tau)_{q s, k}}{\Gamma_{k}(s \beta+\alpha+1)(\zeta)_{p s, k}(\nu)_{s \sigma, k}} \frac{(-2)^{\frac{s}{k}} a^{\frac{s}{k}} b^{\frac{s}{k}}}{a^{\frac{\tau}{k}} b^{\frac{\lambda}{k}}} \frac{k \Gamma_{k}(\tau+s) \Gamma_{k}(\lambda+s)}{\Gamma_{k}(v+\lambda+2 s)} \\
=\frac{\Gamma_{k}(\zeta) \Gamma_{k}(\mu)}{\Gamma_{k}(\tau) \Gamma_{k}(\mu) a^{v} b^{\lambda}} \sum_{s=0}^{\infty} \frac{\Gamma_{k}(\mu+s \rho) \Gamma_{k}(\tau+s q)(-2)^{\frac{s}{k}} a^{\frac{s}{k}} b^{\frac{s}{k}}}{\Gamma_{k}(s \beta+\alpha+1) \Gamma_{k}(\zeta+p s) \Gamma_{k}(\nu+s \sigma) \Gamma} \frac{\Gamma_{k}(v+s) \Gamma_{k}(\lambda+s)}{\Gamma_{k}(v+\lambda+2 s)}
\end{gathered}
$$

we derive required result.

Thus, the proof of Theorem 2.7 is established.

\section{Special Case}

In this section, we establish the following potentially useful integral operators involving generalized k-Beta type functions as special cases of our main results:

(1) If we let $\alpha$ by $\alpha-1$ in Theorem 2.1 and then by using (1.6), we get:

$$
P_{0+}^{(\eta, \lambda)}\left[t^{\frac{\beta}{k}-1} E_{k, \alpha, \beta, \nu, \sigma, \zeta, p}^{\mu, \rho, \tau, q}\left(w t^{\alpha}\right)\right](x)=\frac{x^{\eta+\frac{\beta}{k}} \Gamma\left(\frac{\eta}{(1-\lambda)}+1\right)}{\left(a(1-\lambda)^{\frac{\beta}{k}} k^{\frac{\alpha}{k-1}}\right.} E_{k, \alpha, \beta+k\left(\frac{\eta}{1-\lambda}\right), \nu, \sigma, \zeta, p}^{\mu, \rho, \tau, q}\left(w\left(\frac{x}{a(1-\lambda)^{\frac{\alpha}{k}}}\right)\right.
$$

(2) If we let $\mu=\nu=\sigma=\rho=k=1$ and replacing $\alpha$ by $\alpha-1$ in Theorem 2.1. and then by using (1.7), we obtain:

$$
P_{0+}^{(\eta, \lambda)}\left[t^{\beta-1} E_{\alpha, \beta, p}^{\zeta, \tau, q}\left(w t^{\alpha}\right)\right](x)=\frac{x^{\eta+\beta} \Gamma\left(\frac{\eta}{(1-\lambda)}+1\right)}{(a(1-\lambda))^{\frac{\beta}{k}}} E_{\alpha, \beta+1\left(\frac{\eta}{1-\lambda}\right), p}^{\zeta, \tau, q}\left(w\left(\frac{x}{a(1-\lambda)}\right)^{\alpha}\right)
$$

(3) If we let $\mu=\nu=\sigma=\rho=\zeta=p=1$ and replacing $\alpha$ by $\alpha-1$ in Theorem 2.1 and then by using (1.8), we obtain

$$
P_{0+}^{(\eta, \lambda)}\left[t^{\frac{\beta}{k}-1} E_{k, \alpha, \beta}^{\tau, q}\left(w t^{\alpha}\right)\right](x)=\frac{x^{\eta+\frac{\beta}{k}} \Gamma\left(\frac{\eta}{(1-\lambda)}+1\right)}{\left(a(1-\lambda)^{\frac{\beta}{k}} k^{\frac{\alpha}{k-1}}\right.} E_{k, \alpha, \beta+k\left(\frac{\eta}{1-\lambda}\right), 1,1,1,1}^{1,1, \tau, q}\left(w\left(\frac{x}{a(1-\lambda)}\right)^{\frac{\alpha}{k}}\right)
$$

(4) If we let $\mu=\nu=\sigma=\rho=\zeta=p=k=1$ and replacing $\alpha$ by $\alpha-1$ in Theorem 2.1 and then by using (1.9), we attain:

$$
P_{0+}^{(\eta, \lambda)}\left[t^{\beta-1} E_{\alpha, \beta}^{\tau, q}\left(w t^{\alpha}\right)\right](x)=\frac{x^{\eta+\beta} \Gamma\left(\frac{\eta}{(1-\lambda)}+1\right)}{(a(1-\lambda))^{\beta}} E_{\alpha, \beta+1\left(\frac{\eta}{1-\lambda}\right)}^{\tau, q}\left(w\left(\frac{x}{a(1-\lambda)}\right)^{\alpha}\right)
$$


THE PATHWAY INTEGRAL OPERATOR INVOLVING EXTENSION OF K-BESSEL-MAITLAND FUNCTIOG

(5) If we let $\mu=\nu=\sigma=\rho=q=1$ and replacing $\alpha$ by $\alpha-1$ in Theorem 2.1, and then bu using (1.10), we get

$$
P_{0+}^{(\eta, \lambda)}\left[t^{\beta-1} E_{\alpha, \beta}^{\tau, \zeta}\left(w t^{\alpha}\right)\right](x)=\frac{x^{\eta+\beta} \Gamma\left(\frac{\eta}{(1-\lambda)}+1\right)}{(a(1-\lambda))^{\beta}} E_{\alpha, \beta+1\left(\frac{\eta}{1-\lambda}\right)}^{\tau, \zeta}\left(w\left(\frac{x}{a(1-\lambda)}\right)^{\alpha}\right)
$$

(6) If we let $\mu=\nu=\sigma=\rho=\zeta=p=q=1$ and replacing $\alpha$ by $\alpha-1$ in Theorem 2.1 and then by using (1.11, we attain:

$$
P_{0+}^{(\eta, \lambda)}\left[t^{\frac{\beta}{k}-1} E_{k, \alpha, \beta}^{\tau}\left(w t^{\alpha}\right)\right](x)=\frac{x^{\eta+\frac{\beta}{k}} \Gamma\left(\frac{\eta}{(1-\lambda)}+1\right)}{\left(a(1-\lambda)^{\frac{\beta}{k}} k^{\frac{\alpha}{k-1}}\right.} E_{k, \alpha, \beta+k\left(\frac{\eta}{1-\lambda}\right)}^{\tau}\left(w\left(\frac{x}{a(1-\lambda)}\right)^{\frac{\alpha}{k}}\right)
$$

(7) If we let $\mu=\nu=\sigma=\rho=\zeta=p=q=k=1$ and replacing $\alpha$ by $\alpha-1$ in Theorem 2.1, and then by using 1.12 , we obtain:

$$
P_{0+}^{(\eta, \lambda)}\left[t^{\beta-1} E_{\alpha, \beta}^{\tau}\left(w t^{\alpha}\right)\right](x)=\frac{x^{\eta+\beta} \Gamma\left(\frac{\eta}{(1-\lambda)}+1\right)}{(a(1-\lambda))^{\beta}} E_{\alpha, \beta+1\left(\frac{\eta}{1-\lambda}\right)}^{\gamma}\left(w\left(\frac{x}{a(1-\lambda)}\right)^{\alpha}\right)
$$

(8) If we let $\mu=\nu=\sigma=\rho=\zeta=\tau=p=q=k=1$ and replacing $\alpha$ by $\alpha-1$ in Theorem 2.1, and then by using (1.13), we obtain:

$$
P_{0+}^{(\eta, \lambda)}\left[t^{\beta-1} E_{\alpha, \beta}^{1}\left(w t^{\alpha}\right)\right](x)=\frac{x^{\eta+\beta} \Gamma\left(\frac{\eta}{(1-\lambda)}+1\right)}{(a(1-\lambda))^{\beta}} E_{\alpha, \beta+1\left(\frac{\eta}{1-\lambda}\right)}^{1}\left(w\left(\frac{x}{a(1-\lambda)}\right)^{\alpha}\right)
$$

(9) If we let $\mu=\nu=\sigma=\rho=\zeta=\tau=p=q=k=1, \alpha=0$ and replacing $\alpha$ by $\alpha-1$ in Theorem 2.1, and then by using 1.14, we find:

$$
P_{0+}^{(\eta, \lambda)}\left[t^{\beta-1} E_{\beta}^{1}(w)\right](x)=\frac{x^{\eta+\beta} \Gamma\left(\frac{\eta}{(1-\lambda)}+1\right)}{(a(1-\lambda))^{\beta}} E_{\beta+1\left(\frac{\eta}{1-\lambda}\right)}^{1}\left(w\left(\frac{x}{a(1-\lambda)}\right)\right)
$$

(10) If we let $\alpha$ by $\alpha-1$ in Theorem 2.4. and then by using (1.6), we get:

$$
P_{0+}^{(\eta, \lambda)}\left[t^{\frac{\beta}{k}-1} E_{k, \alpha, \beta, \nu, \sigma, \zeta, p}^{\mu, \rho, \tau, q}\left(w t^{\frac{\alpha}{k}}\right)\right](x)=\frac{x^{\eta+\frac{\beta}{k}+1} \Gamma\left(1-\frac{\eta}{(\lambda-1)}\right)}{\left(-a(1-\lambda)^{\frac{\beta}{k}} k^{\frac{\alpha+1}{k}-1}\right.} E_{k, \alpha, \beta+k\left(n \alpha+k-\frac{\eta}{\lambda-1}\right), \nu, \sigma, \zeta, p}^{\mu, \rho, q}\left(w\left(\frac{x}{-a(\lambda-1)}\right)^{\frac{\alpha}{k}}\right) .
$$

(11) If we let $\mu=\nu=\sigma=\rho=k=1$ and replacing $\alpha$ by $\alpha-1$ in Theorem 2.4. and then by using (1.7), we get:

$$
P_{0+}^{(\eta, \lambda)}\left[t^{\beta-1} E_{\alpha, \beta, p}^{\tau, \zeta, q}\left(w t^{\alpha}\right)\right](x)=\frac{x^{\eta+\beta+1} \Gamma\left(1-\frac{\eta}{(\lambda-1)}\right)}{\left(-a(1-\lambda)^{\beta}\right.} E_{\alpha, \beta+1\left(n \alpha+1-\frac{\eta}{\lambda-1}\right), p}^{\zeta, \gamma, q}\left(w\left(\frac{x}{-a(\lambda-1)}\right)^{\alpha}\right) .
$$

(12) If we let $\mu=\nu=\sigma=\rho=\zeta=p=1$ and replacing $\alpha$ by $\alpha-1$ in Theorem 2.7. and then by using (1.8), we get:

$$
P_{0+}^{(\eta, \lambda)}\left[t^{\frac{\beta}{k}-1} E_{k, \alpha, \beta}^{\tau, q}\left(w t^{\frac{\alpha}{k}}\right)\right](x)=\frac{x^{\eta+\frac{\beta}{k}+1} \Gamma\left(1-\frac{\eta}{(\lambda-1)}\right)}{\left(-a(1-\lambda)^{\frac{\beta}{k}} k^{\frac{\alpha+1}{k}-1}\right.} E_{k, \alpha, \beta+k\left(n \alpha+k-\frac{\eta}{\lambda-1}\right)}^{\gamma, q}\left(w\left(\frac{x}{-a(\lambda-1)}\right)^{\frac{\alpha}{k}}\right) .
$$


(13) If we let $\mu=\nu=\sigma=\rho=\zeta=p=q=k=1$ and replacing $\alpha$ by $\alpha-1$ in Theorem 2.4 and then by using 1.9 , we obtain:

$P_{0+}^{(\eta, \lambda)}\left[t^{\beta-1} E_{\alpha, \beta}^{\tau, q}\left(w t^{\alpha}\right)\right](x)=\frac{x^{\eta+\beta+1} \Gamma\left(1-\frac{\eta}{(\lambda-1)}\right)}{\left(-a(1-\lambda)^{\beta}\right.} E_{\alpha, \beta+1\left(n \alpha+1-\frac{\eta}{\lambda-1}\right)}^{\tau, q}\left(w\left(\frac{x}{-a(\lambda-1)}\right)^{\alpha}\right)$.

(14) If we let $\mu=\nu=\sigma=\rho=q=1$ and replacing $\alpha$ by $\alpha-1$ in Theorem 2.4 and then bu using (1.10), we get

$P_{0+}^{(\eta, \lambda)}\left[t^{\beta-1} E_{\alpha, \beta}^{\tau, \zeta}\left(w t^{\alpha}\right)\right](x)=\frac{x^{\eta+\beta+1} \Gamma\left(1-\frac{\eta}{(\lambda-1)}\right)}{\left(-a(1-\lambda)^{\beta}\right.} E_{\alpha, \beta+1\left(n \alpha+1-\frac{\eta}{\lambda-1}\right)}^{\tau, \zeta}\left(w\left(\frac{x}{-a(\lambda-1)}\right)^{\alpha}\right)$.

(15) If we let $\mu=\nu=\sigma=\rho=\zeta=p=q=1$ and replacing $\alpha$ by $\alpha-1$ in Theorem 2.4 and then by using (1.11), we attain:

$$
P_{0+}^{(\eta, \lambda)}\left[t^{\frac{\beta}{k}-1} E_{k, \alpha, \beta}^{\tau}\left(w t^{\frac{\alpha}{k}}\right)\right](x)=\frac{x^{\eta+\frac{\beta}{k}+1} \Gamma\left(1-\frac{\eta}{(\lambda-1)}\right)}{\left(-a(1-\lambda)^{\frac{\beta}{k}} k^{\frac{\alpha+1}{k}-1}\right.} E_{k, \alpha, \beta+k\left(n \alpha+k-\frac{\eta}{\lambda-1}\right)}^{\tau}\left(w\left(\frac{x}{-a(\lambda-1)}\right)^{\frac{\alpha}{k}}\right) .
$$

(16) If we let $\mu=\nu=\sigma=\rho=\zeta=p=q=k=1$ and replacing $\alpha$ by $\alpha-1$ in Theorem 2.4 and then by using 1.12 , we obtain:

$$
P_{0+}^{(\eta, \lambda)}\left[t^{\beta-1} E_{, \alpha, \beta}^{\tau, q}\left(w t^{\alpha}\right)\right](x)=\frac{x^{\eta+\beta+1} \Gamma\left(1-\frac{\eta}{(\lambda-1)}\right)}{\left(-a(1-\lambda)^{\frac{\beta}{k}}\right.} E_{\alpha, \beta+1\left(n \alpha+1-\frac{\eta}{\lambda-1}\right)}^{\tau, q}\left(w\left(\frac{x}{-a(\lambda-1)}\right)^{\alpha}\right) .
$$

(17) If we let $\mu=\nu=\sigma=\rho=\zeta=\tau=p=q=k=1, \alpha=0$ and replacing $\alpha$

by $\alpha-1$ in Theorem 2.4 , and then by using (1.13), we find:

$$
P_{0+}^{(\eta, \lambda)}\left[t^{\beta-1} E_{\beta}(w)\right](x)=\frac{x^{\eta+\beta+1} \Gamma\left(1-\frac{\eta}{(\lambda-1)}\right)}{\left(-a(1-\lambda)^{\beta}\right.} E_{\beta+1\left(1-\frac{\eta}{\lambda-1}\right)}\left(w\left(\frac{x}{-a(\lambda-1)}\right)^{\alpha}\right) .
$$

(18) If we let $\alpha$ by $\alpha-1$ in Theorem 2.7, and then by using (??), we get:

$$
\begin{aligned}
& \int_{0}^{1} t^{\frac{v}{k}-1}(1-t)^{\frac{\xi}{k}-1}[a t+b(1-t)]^{\frac{-v-\xi}{k}} E_{k, \alpha, \beta, \nu, \sigma, \zeta, p}^{\mu, \rho, q}\left[\frac{-2 a b t(1-t)}{(a t+b(1-t))^{2}}\right]^{\frac{1}{k}} d t \\
= & \frac{\Gamma_{k}(\zeta) \Gamma_{k}(\mu)}{\Gamma_{k}(\tau) \Gamma_{k}(\mu) a^{\tau} b^{\lambda}} \sum_{s=0}^{\infty} \frac{\Gamma_{k}(\mu+s \rho) \Gamma_{k}(\gamma+s q)(2)^{\frac{s}{k}} a^{\frac{s}{k}} b^{\frac{s}{k}}}{\Gamma_{k}(s \beta+\alpha+1) \Gamma_{k}(\zeta+p s) \Gamma_{k}(\nu+s \sigma) \Gamma} \frac{\Gamma_{k}(v+s) \Gamma_{k}(\lambda+s)}{\Gamma_{k}(v+\lambda+2 s)}
\end{aligned}
$$

(19) If we let $\mu=\nu=\sigma=\rho=k=1$ and replacing $\alpha$ by $\alpha-1$ in Theorem 2.7. and then by using (1.7), we get:

$$
\int_{0}^{1} t^{v-1}(1-t)^{\xi-1}[a t+b(1-t)]^{-v-\xi} E_{\alpha, \beta, p}^{\tau, \zeta, q}\left[\frac{-2 a b t(1-t)}{(a t+b(1-t))^{2}}\right] d t={ }_{4} \Psi_{3}\left[\begin{array}{ll}
(\tau, q),(v, 1),(\lambda, 1),(1,1) ; & \\
(\alpha, \beta),(\zeta, p),(v+\lambda, 2), ; & -2 a b
\end{array}\right] .
$$

(20) If we let $\mu=\nu=\sigma=\rho=\zeta=p=1$ and replacing $\alpha$ by $\alpha-1$ in Theorem

2.7 and then by using (1.8), we get:

$$
\int_{0}^{1} t^{\frac{v}{k}-1}(1-t)^{\frac{\xi}{k}-1}[a t+b(1-t)]^{\frac{-v-\xi}{k}} E_{k, \alpha, \beta}^{\tau, q}\left[\frac{-2 a b t(1-t)}{(a t+b(1-t))^{2}}\right]^{\frac{1}{k}} d t
$$


THE PATHWAY INTEGRAL OPERATOR INVOLVING EXTENSION OF K-BESSEL-MAITLAND FUNCTIOIN

$$
=\frac{1}{\Gamma_{k}(\tau) a^{v} b^{\lambda}} \sum_{s=0}^{\infty} \frac{\Gamma_{k}(1+s) \Gamma_{k}(\tau+s q)(2)^{\frac{s}{k}} a^{\frac{s}{k}} b^{\frac{s}{k}}}{\Gamma_{k}(s \beta+\alpha+1) \Gamma_{k}(1+s) \Gamma_{k}(1+s) \Gamma} \frac{\Gamma_{k}(v+s) \Gamma_{k}(\lambda+s)}{\Gamma_{k}(v+\lambda+2 s)}
$$

(21) If we let $\mu=\nu=\sigma=\rho=\zeta=p=k=1$ and replacing $\alpha$ by $\alpha-1$ in Theorem 2.7 and then by using (1.9), we attain:

$$
\left.\int_{0}^{1} t^{v-1}(1-t)^{\xi-1}[a t+b(1-t)]^{-v-\xi} E_{\alpha, \beta}^{\tau, q}\left[\frac{2 a b t(1-t)}{(a t+b(1-t))^{2}}\right] d t={ }_{3} \Psi_{2}\left[\begin{array}{c}
(\tau, q),(v, 1),(\lambda, 1) ; \\
(\alpha, \beta),(v+\lambda, 2), ;
\end{array}\right] .2 a b\right] .
$$

(22) If we let $\mu=\nu=\sigma=\rho=q=p=k=1$ and replacing $\alpha$ by $\alpha-1$ in Theorem 2.7 and then by using (1.10, we attain:

$$
\left.\int_{0}^{1} t^{v-1}(1-t)^{\xi-1}[a t+b(1-t)]^{-v-\xi} E_{\alpha, \beta}^{\tau, \zeta}\left[\frac{2 a b t(1-t)}{(a t+b(1-t))^{2}}\right] d t={ }_{3} \Psi_{3}\left[\begin{array}{c}
(\tau, 1),(v, 1),(\lambda, 1) ; \\
(\alpha, \beta),(v+\lambda, 2),(\zeta, 1), ;
\end{array}\right] .2 a b\right] .
$$

(23) If we let $\mu=\nu=\sigma=\rho=\zeta=p=q=1$ and replacing $\alpha$ by $\alpha-1$ in Theorem 2.7 and then by using (1.11), we attain:

$$
\begin{aligned}
& \int_{0}^{1} t^{\frac{v}{k}-1}(1-t)^{\frac{\xi}{k}-1}[a t+b(1-t)]^{\frac{-v-\xi}{k}} E_{k, \alpha, \beta}^{\tau}\left[\frac{-2 a b t(1-t)}{(a t+b(1-t))^{2}}\right]^{\frac{1}{k}} d t \\
= & \frac{1}{\Gamma_{k}(\tau) a^{v} b^{\lambda}} \sum_{s=0}^{\infty} \frac{\Gamma_{k}(1+s) \Gamma_{k}(v+s)(2)^{\frac{s}{k}} a^{\frac{s}{k}} b^{\frac{s}{k}}}{\Gamma_{k}(s \beta+\alpha+1) \Gamma_{k}(1+s) \Gamma_{k}(1+s) \Gamma} \frac{\Gamma_{k}(v+s) \Gamma_{k}(\lambda+s)}{\Gamma_{k}(v+\lambda+2 s)} .
\end{aligned}
$$

(24) If we let $\mu=\nu=\sigma=\rho=\zeta=p=q=k=1$ and replacing $\alpha$ by $\alpha-1$ in Theorem 2.7 and then by using (1.12), we obtain:

$$
\left.\int_{0}^{1} t^{v-1}(1-t)^{\xi-1}[a t+b(1-t)]^{-v-\xi} E_{\alpha, \beta}^{\tau}\left[\frac{-2 a b t(1-t)}{(a t+b(1-t))^{2}}\right] d t={ }_{3} \Psi_{2}\left[\begin{array}{c}
(\tau, 1),(v, 1),(\lambda, 1) ; \\
(\alpha, \beta),(v+\lambda, 2), ;
\end{array}\right] .2 a b\right] .
$$

(25) If we let $\mu=\nu=\sigma=\rho=\zeta=\tau=p=q=k=1$ and replacing $\alpha$ by $\alpha-1$ in Theorem 2.7, and then by using (1.13) we obtain

$$
\left.\int_{0}^{1} t^{v-1}(1-t)^{\xi-1}[a t+b(1-t)]^{-v-\xi} E_{\alpha, \beta}\left[\frac{-2 a b t(1-t)}{(a t+b(1-t))^{2}}\right] d t={ }_{3} \Psi_{2}\left[\begin{array}{c}
(1,1),(v, 1),(\lambda, 1) ; \\
(\alpha, \beta),(v+\lambda, 2), ;
\end{array}\right] .2 a b\right] .
$$

(26) If we let $\mu=\nu=\sigma=\rho=\zeta=\tau=p=q=k=1, \alpha=0$ and replacing $\alpha$ by $\alpha-1$ in Theorem 2.7, and then by using 1.14 we obtain:

$$
\left.\int_{0}^{1} t^{v-1}(1-t)^{\xi-1}[a t+b(1-t)]^{-v-\xi} E_{\beta}\left[\frac{-2 a b t(1-t)}{(a t+b(1-t))^{2}}\right] d t={ }_{3} \Psi_{2}\left[\begin{array}{c}
(1,1),(v, 1),(\lambda, 1) ; \\
(0, \beta),(v+\lambda, 2), ;
\end{array}\right] .2 a b\right] .
$$

\section{Conclusion}

In the present article, we derive a new generalization of k-Beseel Maitland function and obtain the fractional calculus formula for the same. We also define and study a new fractional integral operators, which contain the extended Bessel Maitland 
function. If $k=0$, then all the results of extended Bessel Maitland function will lead to the well-known results of Bessel Maitland function (see [9]).

Acknowledgments. The authors are very grateful to the referee for many valuable comments and suggestions which helped to the improve the paper.

\section{REFERENCES}

[1] Agarwal, P; Purohit, S. D . The unified pathway fractional integral formulae, Fract. Calc. Appl., 4(9)(2013), 1-8.

[2] Bairwa, R. K; Sharma, S. C. Certain properties and integral transforms of the k-Generalized Mittag-leffler type function, J. Int. Acad. Physical Sciences, 19(4)(2015), 277-294.

[3] Chaudhry, M. A; Qadir, A; Rafiq, M; Zubair, S. M. Extension of Euler's Beta function, J. Comput. Appl. Math., 78(1)(1997),19-32.

[4] Chand, M., Prajapati, J.C., Bonyah, E. Fractional integral and solution of fractional kinetic equation involving k-MittagLeffler function. Trans. A. Razmadze Math. Inst. 171(2017), 144166 .

[5] Choi, J; Agrawal, P. Certain unified integrals associated with special functions, Boundary Value Problems, Vol. 2013(2013),(95).

[6] Dorrego, G. A; Cerutti, R. A. The k-Mittag-Leffer function, Int. J. Contemp. Math. Sci., 7 (2012), 705-716.

[7] Ghayasuddin, M; Khan, W. A; Araci, S. A new extension of Bessel Maitland function and its properties, Matematicki, Vesnik, Mathe. bechnk, 70(4)(2018), 292-302.

[8] Khan, M. A., Ahmed, S. On some properties of the generalized Mittag-Leffler function, Springer Plus, 2:(2013),337.

[9] Khan, A. W; Khan, A. I; Ahmad, M. On Certain integral transforms involving generalized Bessel-Baitland function, J. Appl. Pure

Math., 2 (1-2)(2020), 63-78.

[10] Luke, Y. L. The Special functions and their approximations,vol.1, New York, Academic Press 1969.

[11] MacRobert, T. M. Beta functions formulae and integral involving E-function Math. Annalen, $142(1961), 450-452$.

[12] Marichev, Ö. I. Handbook of integral transform and higher transcendental function. Theory Algorithm Tables, Ellis Horwood, Chichester [ John Wiley and sons], New York 1983.

[13] Mathai, A. M. A pathway to matrix-variate gamma and normal densities, Linear Algebra Appl., 396(2005),317-328

[14] Mathai, A. M; Haubold, H. J. Pathway model super statistics, trellis statistics and generalized measure of entropy, Phys. A. 375(2007), 110-122.

[15] Mathai, A. M; Haubold, H. J. On generalized distributions and pathways phys. LCH. A. 372(2008), 2019-2113.

[16] Mittag-Leffler, G. M, and Sur la. Nouvelle function $E_{\alpha}(x)$, C. R .Acad. Sci Paris, 137(1903), 554-558. 
THE PATHWAY INTEGRAL OPERATOR INVOLVING EXTENSION OF K-BESSEL-MAITLAND FUNCTIOR

[17] Nair, S. S. Pathway fractional integral operator, Fract. Calc. Appl, Anal, 12(3)(2009), 237252.

[18] Nisar, K. S; Mondal, S. R. Pathway fractional integral operators involving k-Struve function, arXIV: 1611;(2016) 09157[math. C. A].

[19] Nisar, K. S; Mondal, S. R; Agraval, P. Pathway fractional integral operator associated with Struve function of first kind, Advanced Studies Contemporary Math. 26(2016),63-70.

[20] Nisar, K. S; Eata, A. F; Dhatallah, M; Choi, J. fractional calculus of generalized k-MittagLeffler function and its application, Adv. Diff. Equations, 1(2016), 304.

[21] Rainville, E. D. Special functions, The Macmillan Company, New York 1960.

[22] Prabhakar, T. R. A singular integral equation with a generalized Mittag-Leffler function in the kernal, Yokohama Math. J., 19(1971), 7-15.

[23] Salim, T. O; Faraj, W. A generalization of Mittag-Leffler function and integral operator associated with fractional calculus, Appl. Math. Comput., 3(5)(2012),1-13.

[24] Salim, T. O. Some properties relating to the generalized Mittag-Leffler function, Adv. Appl. Math. Anal., 4(2009), 21-80.

[25] Singh. M; Khan. M; Khan A. H. On some properties of a generalization of Bessel Maitland function, Int. J. Math. Trends Tech., 14(1)(2016), 46-54.

[26] Shukla, A.K; Prajapati, J. C. On a generalization of Mittag-Leffler function and its properties, J. Math. Anal. Appl., 336(2007), 797-811.

[27] Watson, G. N. A treatise on the theory of bessel functions, Cambridge University Press 1962.

[28] Wiman, A. Uber den fundamental satz in der theory der funktionen, Acta Math., 29(1905), 191-201.

[29] Wright, E. M. The asymptotic expansion of the generalized hypergeometric function, J. Lond. Math. Soc, 10(1935), 286-293.

[30] Wright, E. M. The asymptotic expansion of integral functions defined by Taylor series, Philos. Trans. R. Soc. Lond. A, 238(1940), 435-451.

[31] Wright, E. M. The asymptotic expansion of the generalized hypergeometric function II, Proc. Lond. Math. Soc, 46(1940), 389.

Moin AHmad,

Department of Mathematics, Shree Krishna Educational Institute, Ahmad Nagar Sitapur261125, (U.P.), INDIA, 9807480087, ORCID ID: 0000-0002-1482-8197

Email address: moinah1986@gmail.com

SAurabh Porwal,

Department of Mathematics, Ram Sahai Government Degree College, Bairi-Shivrajpur, KANPuR-209205, (U.P.), India, 9415937193, OrCID ID: 0000-0003-0847-3550

Email address: saurabhjcb@rediffmail.com 